\title{
Faculty-Student Collaboration: Issues and Recommendations
}

\author{
Angeline Barretta-Herman \\ Kendra J. Garrett
}

\begin{abstract}
This exploratory qualitative study of 11 social work faculty identified the benefits and risks of faculty-student collaboration. Benefits articulated include helping students learn to write for publication, learning the publication process, getting innovative student material published, and enriching the project through shared problem-solving. The benefits, however, must be weighed against the risks of exploitation of the student collaborator. Successful faculty-student collaboration in this dual relationship demands that faculty take responsibility for safeguarding boundaries, following the NASW Code of Ethics, and openly negotiating roles, tasks, workload, and order of authorship with the student.
\end{abstract}

Keywords: Faculty-student, collaboration, mentor, multiple authorship, dual relationship, publication ethics

\begin{abstract}
A cademia encourages faculty-student collaboration in research and publication as a strategy for creating a mentoring relationship and providing students with a valuable opportunity to gain experience in working with a proven researcher (Austin \& Baldwin, 1991). Nevertheless, there are risks that must be acknowledged when forming faculty-student collaborations (Congress, 1996). The NASW Code of Ethics (NASW, 1996) prohibits dual relationships in academia when there is a "risk of exploitation or potential harm to the student" (Section 3.02 (d), p. 19). Students are dependent on faculty for expertise, grades, and references, leaving them in a less powerful position (see Leatherman, 1997). The power differential inherent in the faculty-student relationship carries over into the collaborative relationship (Kagel \& Giebelhausen, 1994). Bonosky (1995) suggests that educators have a fiduciary accountability to protect students who are dependent on the knowledge and skills of the faculty. Thus, it is the faculty's responsibility to set clear boundaries and protect students from exploitation. This is particularly pertinent when the student works with faculty on a research project that could potentially lead to publication or co-authorship. Because of these concerns, the authors conducted exploratory qualitative research to learn the perceptions of social work faculty who had participated in faculty-student collaborations. This was viewed as a critical first step toward developing ethical guidelines.
\end{abstract}

Angeline Barretta-Herman, Ph.D. is Professor and M.S.W. Program Director, and Kendra J. Garrett, D.S.W. is Assistant Professor, School of Social Work, College of St. Catherine/University of St. Thomas, St. Paul, Minnesota, USA.

Copyright@ 2000 Advances in Social Work Vol. 1 No. 2 (Fall 2000) 148-159 .

Indiana University School of Social Work. 


\section{REVIEW OF THE LITERATURE}

Faculty-student collaboration as the major focus (Fine \& Kurdek, 1993; Netting \& Nichols-Casebolt, 1997; Over, 1982) or as part of discussions on collaboration (Austin \& Baldwin, 1991; Krysik \& Nichols-Casebolt, 1994), mentorship (Wright \& Wright, 1987), and multiple authorship (Gelman \& Gibelman, 1999; Zook, 1987) has received limited attention across the disciplines. Discussions of collaboration and coauthorship between colleagues of equal or unequal rank has dominated the literature.

\section{Benefits of Collaboration}

Several benefits to collaboration are noted in the literature. Aram, Morgan, and Esbeck (1971) assert that collaboration promotes psychological well-being by penetrating the barrier of academic isolation. Collaboration can assist the student or academic neophyte to negotiate the maze toward publication (Wright \& Wright, 1987). For colleagues, collaboration can serve to generate ideas, maintain momentum, emphasize individual expertise, and provide opportunity for faculty to develop new skills (McCullagh, 1988).

Research by Gordon (1980), Presser (1980), and Endersby (1996) suggests that collaboration is particularly beneficial when conducting interdisciplinary research. Zook (1987) notes that the challenge to remain current in this period of exponential knowledge and methodological development demands collaboration among colleagues because "the chances that a given individual can maintain currency with a wide spectrum of fields is increasingly. . remote" (p. 78).

\section{Impact on Tenure and Promotion}

The literature reports wide variations in academia regarding the relative importance of solo, dual, and multiple authorship. Bayer and Smart (1991) note that the biomedical fields and "hard sciences" are highly collaborative in stark contrast to the humanities, which have "markedly lower rates of collaboration" (p. 613). They suggest that a biomedical scientist with a solo pattern of publication might be considered suspect when compared to a colleague with a collaborative pattern. The latter colleague is likely to be viewed as a "team player" (p. 614)—able to work with colleagues, learn from them, and expose his or her work to critical commentary. Endersby (1996) concluded that "collaboration in the physical sciences is the norm... and the number of credited authors is increasing in most disciplines" (p. 376).

In the humanities where work in libraries and archives is performed independently, collaboration is less common. Here, the primacy of solo scholarship is most apparent in tenure and promotion decisions. Collaboration for developing scholars can be particularly detrimental in settings where solo authorship is deemed supreme (Austin \& Baldwin, 1991; Fox \& Favor, 1984). The case is much the same in the social work profession. Netting and Nichols-Casebolt's (1997) respondents agreed that "sole authorship is important for tenure and promotion decisions in schools of social work. Single authorship demonstrates one's ability to do independent scholarship" (p. 562).

\section{Author Order}

Bayer and Smart's (1991) research on author order in multiple authorship highlights the wide variations in protocol. In some fields, senior faculty traditionally 
take the first author position. However, Martin's study (cited in Bayer \& Smart, 1991) noted the "reverse snobbery" practiced in selected disciplines that place the senior faculty last. "Authorship order is not entirely a function of just individual attitudes. It is also a reflection of differences in disciplinary practices" (p. 616). Endersby (1996) highlighted discipline-based protocols. He found that although social sciences most often cite multiple authorship in alphabetical order, there are dramatic differences within the social sciences. "Four-fifths of economists list authors alphabetically, only a third of psychologists do" (p. 382).

The American Psychological Association (1992) published ethical standards for reporting and publishing of scientific information. The standards mandated that psychologists should not present the material of others as their own, nor credit others who did not contribute to the work. Psychologists were advised that "Mere possession of an institutional position... does not justify authorship credit" (Section 6.23(b), p. 1609). With regard to faculty-student collaboration, the standards state that a student is to be principal author on any article that is "substantially based on the student's dissertation or thesis" (Section 6.23 (c), p. 1609). The Social Work Code of Ethics (NASW, 1996) concurs regarding acknowledging the credit of others. "Social Workers should take responsibility and credit, including authorship credit, only for work they have actually performed and to which they have contributed" (Section 4.08 (a), p. 24).

\section{The Challenges of Collaboration}

The potential conflicts in assigning credit, identifying the origin of the idea, negotiating author order, and determining "substantial credit" can be a "matter of individual perspective" (Floyd, Schroeder, \& Finn, 1994, p. 744). The APA Publication Manual (1994) attempts to distinguish between substantial professional contribution to a work (formulating the problem or hypothesis, structuring the experimental design, organizing and conducting the statistical analysis, interpreting the results, or writing a major portion of the paper) and lesser supporting contributions for which only acknowledgment might be necessary (such as suggesting or advising about the statistical analysis, collecting or entering the data, and recruiting participants). However, the challenge of distinguishing between substantial and supporting contributions is widely acknowledged, and that distinction may become more or less clear over the life of the project.

It is important to explore understanding of this complex issue for three reasons. First, discussion of faculty-student collaboration in social work is in its initial stage. The lack of research, literature, and guidelines is acknowledged in the literature (Gibelman \& Gelman, 1999). Second, encouraging faculty-student collaboration is one means to prepare "the next generation of social work scholars" (Netting \& NicholsCasebolt, 1997, p. 563). And lastly, the social work profession has been challenged to increase research productivity (Task Force on Social Work Research, 1991). Through mentoring, faculty-student collaborations may provide a way to make student scholarly work available to the professional community and support faculty scholarship.

\section{METHODOLOGY}

The authors chose a qualitative research design due to the exploratory nature of the research and their desire to gain in-depth understanding of the respondents' 
experiences. Gibelman and Gelman (1999) point out the improbability of gathering reliable data on unethical practices through survey research. Using a modified snowball sampling technique, the authors sought to draw a regionally representative sample of social work faculty who had experience being in a faculty-student collaborative relationship either as faculty or students. As Table 1 indicates, 11 taped interviews were conducted with faculty from all parts of the country. A respondent gender balance of five women and six men was achieved. Faculty rank was unevenly balanced among the three ranks of assistant, associate, and full professor, with six of the 11 respondents holding the assistant professor rank. Although half the respondents are presently in teaching institutions, eight described collaborative relationships that took place in research institutions.

\begin{tabular}{|l|l|l|l|l|l|l|c|}
\hline \multicolumn{2}{|l|}{ Table 1: Demographics } \\
\hline $\begin{array}{l}\text { Respon- } \\
\text { dent }\end{array}$ & Gender & Rank & Location & $\begin{array}{l}\text { Position } \\
\text { as } \\
\text { Collabo- } \\
\text { rator for } \\
\text { This } \\
\text { Research }\end{array}$ & $\begin{array}{c}\text { Kind } \\
\text { of } \\
\text { Insti- } \\
\text { tution }\end{array}$ & $\begin{array}{l}\text { Years } \\
\text { Since } \\
\text { Collabo- } \\
\text { ration }\end{array}$ & $\begin{array}{c}\text { Gender of } \\
\text { Collaborator(s) }\end{array}$ \\
\hline 1 & F & Asst. & South & Student & Research & 10 & F \\
2 & M & Asst. & Midwest & Student & Research & 3 & M \\
3 & M & Full & Midwest & Faculty & Research & 2 & M,F \\
4 & F & Assoc. & West & Faculty & Teaching & 2 & M \\
5 & F & Full & Southwest & Faculty & Research & 2 & F \\
6 & M & Asst. & East & Student & Research & 5 & M \\
7 & M & Asst. & Northwest & Student & Research & 10 & F \\
8 & F & Full & East & Faculty & Research & 1 & F \\
9 & M & Asst. & Midwest & Student & Teaching & 20 & M \\
10 & F & Assoc. & Midwest & Faculty & Teaching & 5 & F \\
11 & M & Asst. & Southeast & Student & Research & 2 & M \\
\hline
\end{tabular}

Respondents were initially contacted by phone with a description of the research. If they agreed, a consent letter was sent describing the types of questions they would be asked and detailing the arrangements for the interview. Respondents were asked to describe their experience in a faculty-student collaboration of their choosing. The researchers did not intervene in that choice but asked only that the respondents choose an experience with collaboration details they could recall and an experience they wanted to discuss. It is important to note that of the six student collaborations that respondents chose to describe, three occurred 10 or more years ago.

The respondents were asked questions regarding the process, including workload decision-making, determining authorship order, satisfaction with the process, other collaborative work, and recommendations for successful collaboration. Five face-to-face interviews were conducted at a place of the respondents' 
choosing; the rest were conducted using a speakerphone in order to audiotape the interviews. The tapes were transcribed and independently analyzed to identify categories and themes, employing standard manual content analysis techniques.

Confidentiality was of major concern to several of the respondents who expressed concern that the details of their experience, if reported accurately, would unnecessarily embarrass or anger their collaborators. The researchers carefully selected quotes and described the reported experiences. To protect the confidentiality of the respondents, drafts of the finished manuscript were sent to all respondents for review. Respondents were asked to review and comment on the manuscript. No respondent requested that any reference or description be altered or excised. Sending the manuscript for review had a second, but equally important function. Respondents' review and comment on the manuscript enhanced its accuracy and validity.

\section{RESULTS}

Although all of the respondents currently hold a faculty position, six were students at the time of the collaboration. They are described below as "student collaborators." Those who were faculty at the time of the collaboration are labeled "faculty collaborators."

None of the respondents' schools had policies, formal or informal, about facultystudent collaboration for publication. One faculty collaborator indicated that final projects were viewed as an opportunity for such collaboration, but faculty were left on their own to determine how that collaboration was to occur. Three respondents indicated that they had created their own rules to guide the process. One discussed being very clear about the equality of authorship credit and described her experience as an application of a feminist model. Another indicated that the student is always the first author in a collaborative effort between herself and a student.

\section{Decision Making and Planning}

Faculty members, rather than students, initiated most of the reported collaborative efforts. There was a beginning phase in which faculty chose students with whom they felt comfortable and who were interested in similar topics:

As she and I worked on developing [a classroom assignment], I really was impressed with the work on it and we talked about the possibility that maybe we would work together to publish it when she finished the course. After she finished her assignment and graduated, we worked on it over the summer and it became published.

Faculty viewed the students with whom they collaborated as among their best students:

I got involved with [the students] because they had been with my advanced research class... The students at [location] were extremely bright, hard-working people. I could have picked any one of 20 students in that research class, but I picked these three women.

The faculty initiated discussions about roles, tasks, responsibilities, or order of authorship before the collaboration began. Three faculty collaborators indicated that they felt it was important to discuss roles and expectations and the way the collaboration would take place before deciding to work together. When no discus- 
sion about the collaborative process occurred, student collaborators assumed that the faculty collaborator would assign tasks. They felt humbled by the prospect of writing a professional paper for publication, were pleased to be involved, and rarely thought about authorship issues. Some described themselves as naive about how collaboration generally takes place and expressed a wish that there had been a discussion about the collaborative processes, what was expected, and how authorship would be determined. One student collaborator said:

There was never any discussion about tasks and who was going to do them other than that the two experienced faculty members were going to do the majority of the work. So, for the most part, I didn't do any of the writing. I was wondering about that, but I wasn't assertive about it, and I wasn't clear about what I should be doing.

One faculty collaborator used what she described as a feminist model in writing, in which collaborators shared the work but did not, in her words, "worry about the amount" of each person's contribution. Authorship order in this case was alphabetical. The planning done by this group was an ongoing process.

Some people felt much more comfortable with the majority of the literature search and literature review. Other people had other strengths. It was a process we went through as we worked through and sometimes shifts would be made. . It was always a process. . . always in motion, which might drive some people nuts.

\section{The Collaboration Process}

Respondents described a broad range of activities under the term "collaboration." Some shared responsibility equally, others had a relationship in which one person (usually the faculty collaborator) took major responsibility for assigning roles and tasks, one did virtually all of the work, and others described shifting responsibilities. One faculty respondent advocated for a partnership involving compromise and shared ownership:

When you are collaborating, you spell out and develop and reach consensus on what the project is so that everybody has ownership. So, if I come to you with a project, we have to massage that and incorporate your ideas so that we're really looking at something that's the whole. We talk about shared power; we talk about how decisions are going to be made; we talk about how conflict is going to be resolved. I think that it's really a collaboration when two people come together and give up some pieces. .. When we both come with our distinct roles and they don't at some point merge, then, that's not collaboration... You've got to have something that everybody owns.

Respondents also described a wide variety of working styles. Some would meet regularly to compare notes and combine what they had written separately. One pair sat together in front of a word processor and composed simultaneously. Some wrote independently, then blended the parts together. Most described meetings in which joint work was discussed either before or after it had been entered into a document. One student collaborator who felt less satisfied by the collaborative effort described reporting to the first author who did not reciprocate by sharing information. 


\section{Power Issues}

Four faculty collaborators indicated a desire to work together and encourage students to work with them as equals or partners. They acknowledged that this was difficult at first, as students were accustomed to viewing the faculty person as an evaluator. Initially, even when faculty members were working toward a sense of partnership, students were hesitant to challenge them. "I think the issue of power became problematic ... because I was bending so hard over backwards not to be using power. . I felt a little bit taken advantage of."

One faculty collaborator indicated a sense of responsibility to set guidelines or boundaries because of the power differential:

I am always very clear that I'm the professor with the student, the authority. And with a [MSW] graduate, there hasn't been much of a [power] shift. With a doctoral student, the shift comes fairly quickly that they [move] to a co-equal status before they begin [the collaboration].

Student collaborators also commented on the need for equality to feel a true sense of collaboration. One felt mentored by his faculty person who encouraged him to express his ideas and take ownership of the project. Others did not feel their faculty collaborator shared power with them. They were continuously aware of their lower status and aware of the extent of their dependency on the faculty person, "I did feel very vulnerable during the whole process. I felt subservient. . . [I] definitely felt a sense of hierarchy and a certain amount of fear that if I didn't play the game right I wouldn't get my Ph.D."

Of the six student collaborators interviewed, five felt that the faculty person with whom they collaborated had exploited them in some way. These former students talked about how they felt betrayed by someone they trusted to look after their interests. Four spoke with anger and a sense of pain because of what they perceived as a clear misuse of power.

I did all the research on it and wrote up the paper, pretty much the whole thing, and then we got back together and recommendations were made about how it could be changed, and then I would go back and change it because I was the one who had it on the computer.. But it finally came after multiple revisions... to a version that we sent out to a publisher and basically it went out with my name second on it.

It is important to note that none of these student collaborators had communicated their anger or concern to their faculty collaborator. Two expressed concern that their faculty collaborator not be identified or learn that they had participated in this research. Two others indicated that they knew that their faculty collaborators had published their material without giving the student collaborator credit because colleagues had informed them of it. However, neither had personally looked up the published article and both expressed a reluctance to do so.

I never tried to find [the article published by my professor]. I think that I was so stunned by it all that I just kind of went on. I think that basically I was in a very vulnerable place personally at that time. I think the reason that I didn't try to find out more about it is that I didn't have the psychological strength to deal with it because it felt like it was going to be a conflict. 


\section{Costs of Collaboration}

Student collaborators talked about the process as an exchange. They gave up some independence and solo authorship in exchange for help from the faculty person and experience in the publication process. "At the time I don't think I did [feel taken advantage of]. Now I do. But, like I said, at the time... I was fairly happy to be getting what I wanted."

Three former student collaborators still feel confusion about the collaborative process, and especially about feelings towards their former faculty collaborators. They questioned their own feelings and judgment. They wondered how responsible they were for having been taken advantage of by the person in power. One student collaborator spoke about the internal contradiction he felt towards his former mentor, indicating that he felt a fondness for the mentor while at the same time knowing the mentor was using him. He indicated that the confusing boundaries of that relationship still haunt him many years later. Another student collaborator doubts his ability to read the situation:

Part of me says that I don't have a policy, nothing to go on, so, is it just me? Or, is this really unethical? That's a big part of why I hesitate. There isn't anything to compare what I see has occurred and what's appropriate... I guess I'm kind of torn and ambivalent about it. On the one hand, I'm irritated by it because of all the work I put in. On the other hand, I don't know. I don't have anything to compare it with-whether this was O.K. to do.

Another cost of a poorly structured collaborative relationship between faculty and student merits mention. Two of the student collaborators who felt they had been exploited by a faculty collaborator indicated that they are now reluctant to enter into faculty-student collaborations: "I think that I've been pretty hesitant to [collaborate with students]. I haven't sought it out...And, I hadn't really thought about why not. . . Maybe because of my bad experience, I worry about the boundary issues."

\section{Perceptions of Other Students}

Three respondents mentioned reactions from other students regarding the facultystudent collaboration. One student collaborator indicated that his fellow students were quite accepting of the collaboration and expressed a wish that they could also find such a mentoring relationship. A faculty collaborator indicated that she always waits until after a student has graduated before initiating a collaborative project so that the collaboration does not affect relationships in the classroom. Another expressed a desire to keep the collaboration quiet:

I'm not sure that other students were aware of [the collaboration] and I think that it was [deliberate], because how could I explain that these nine were part of it and others weren't? It was a matter of hurt feelings or feeling like maybe they weren't liked. So, I'm not sure that I made other students that widely aware of it. It was a sticky piece. . I really didn't want other students to know about it.

\section{Faculty as Mentor}

Faculty collaborators assume part of their role is to mentor students and help them learn to publish. They spoke of the satisfaction in helping students move 
beyond the student role and become contributors to the knowledge base of the profession. "I felt like I had a part in getting something important into the literature. I really felt like it was a wonderful, important study. That was fun and I liked the feeling of nurturing somebody." Another said, "My goal is ... to help them really learn how [to publish] ... so I see it as an apprenticeship learning."

Others spoke of the responsibility of faculty, once they are established in their own careers, to help others become established as well.

That is kind of the role I see myself in as a faculty member. . to help develop people's capacities and skill to a point where they demonstrate it ... through some type of publication in a scholarly journal.

A student collaborator indicated gratitude for the willingness of a senior faculty person who shared time, effort, and first author status to help him begin his writing career: "I felt very honored just to be working with somebody with that stature. Somebody who has all those skills but is very willing to give-it's a gift."

Student collaborators expressed their desire for help in learning how to publish, and some described experiences in which they received it:

I did write another paper. . [and] some professors looked at it. One professor in particular, worked with it, helped me, taught me how you do it, and that paper got published. And, I was sole author on it. He did not put his name on it at all, but that paper was a published paper. I think that I learned a lot from him in terms of how you can help students with that.

\section{Collaboration in Social Work}

Respondents expressed the opinion that collaborative work was valuable and a model toward which to strive. They indicated a satisfaction and enjoyment they had with collaboration, which was not there when they worked on their own:

I think that one of the major advantages that I see in it is that it makes the project richer because you have two minds or more working. I think it becomes richer and more valuable because it's not just one person's thinking going on. I also think that it's a healthful model for what we should be doing more of, collaborating with each other. Not only within the university, but out in the community.

Despite the idea that social workers can benefit from collaborative work, two respondents indicated that their institutions regarded collaborative work as substandard, particularly for purposes of promotion and tenure.

\section{DISCUSSION}

The respondents described a wide variety of experiences that ranged on a continuum from equal contribution and credit for theory development, literature review, data collection, writing, and overall management of the project at one extreme, to student collaborators who performed most or all of the work and received no credit or acknowledgement at the other extreme. All respondents acknowledged their commitment to assume responsibility for setting boundaries and safeguarding student rights when working collaboratively with them. Of the six student respondents who were involved in faculty-student collaboration, five felt exploited in 
some way by their faculty collaborators. The pain and anger of their experience remains despite the passage of a considerable amount of time. Their negative experience constrains the way these former students, now faculty themselves, work with students. Two hesitate to collaborate with students. All of the student collaborators who described negative experiences also discussed a subsequent successful collaboration with colleagues. Although this positive collaborative experience gave them a model for appropriate, non-abusive collaboration, the memory of the negative experience remains strong.

It is especially important to recognize the mixed and ambivalent feelings that five of the student collaborators reported. They felt both beholden to and taken advantage of by the person they trusted as a mentor. These mixed feelings led to a sense of self-doubt that has been extremely slow to resolve. This parallels the dynamics of other boundary violations in which the victim was unsure whether or not he/she was the responsible party. It underscores faculty responsibility to safeguard boundaries and avoid student exploitation. Two respondents expressed a lingering concern that their participation not be identifiable to their former mentors.

It is also worth noting that three of the faculty collaborators who were interviewed were aware of these boundary issues and had created their own set of rules for collaborating with students. They viewed collaboration with students as a part of their responsibility to ensure that future social work scholars will continue to contribute to the knowledge base of the profession.

There are recognizable limitations to this study. It is not possible to generalize about faculty-student collaborations, as it was an exploratory study with a small sample. It is possible that the subjects chose to elaborate on their negative experiences rather than their positive ones because the negative experiences stand out for them or because they perceived that the researchers were more interested in their negative stories. Nevertheless, the concerns and issues raised by these respondents highlight the risks of dual relationships in faculty-student collaboration. If faculty do not take responsibility for safeguarding student rights in collaborative relationships, students are likely to be at risk for exploitation.

\section{RECOMMENDATIONS}

One: It is imperative that social work, as a discipline, engage in deliberate, focused, frank, and open discussion regarding the value of collaborative efforts and the regard attributed to them in academia. Social work educators must address the extent to which collaboration and the synergy of small group scholarship efforts should be recognized and respected. Only then can the issue of the relative value of solo versus co- or multiple authorship in tenure and promotion decisions be addressed.

Two: Deans and directors must take responsibility for initiating discussions with faculty to develop protocols that guide faculty-student collaborations. The experiences reported in this study raise several disturbing issues that can best be addressed by thorough discussion and full understanding of the complexity of collaboration, in general, and faculty-student collaboration, in particular. 
Three: The faculty member in faculty-student collaborations carries the primary responsibility for avoiding the risk of exploitation. Professional responsibility is clearly delineated in the NASW Code of Ethics (NASW, 1996). Initial discussions about each collaborator's responsibilities, authorship order, and credit about workload, tasks, and responsibilities must be followed by periodic review of those expectations and contributions with modifications to the original agreement, as required.

Four: Collaboration can be enhanced by written agreements. Making those agreements known to others can minimize the risk of exploitation. Faculty-student collaboration agreements reviewed in consultation with non-collaborating colleagues, deans, or directors can provide an opportunity to forestall future misunderstandings and enhance the development of non-exploitative guidelines.

This study raises several difficult issues that must be researched before firm guidelines can be established. The ethical implications of multiple authorship are coming under scrutiny (see, for example, Gelman \& Gibelman, 1999). Not enough is known about the processes and myriad implications that collaborations have for the individuals involved. Replication of this study with a random sample of social work educators will confirm or refute the findings of this study. Other questions need to be answered: Does faculty-student collaboration lead to increased dissemination of student research? How can "substantial contribution" to a project be evaluated? How should changes in responsibility be renegotiated over the course of collaboration? How do the perceptions of collaboration differ in the faculty-student dyad? What is the ideal protocol for authorship order? What approach to multiple authorship best serves the social work profession? Is the absence of program and institutional protocol, as evidenced in this study, the result of default or design? Are the products of faculty-student collaboration valued in social work programs and the academic institution?

We are convinced that discussion must occur regarding the faculty-student collaboration issues raised here. Our profession has an opportunity to maximize the benefits that accrue through collaboration, including helping students learn to write for publication, getting innovative student material published, and enriching projects through shared problem solving. While these are advantageous to the students, they also benefit faculty, the profession, and ultimately those we serve. Social work is a profession that espouses the virtues of collaborative and cooperative efforts and emphasizes the importance of a systemic view of phenomena. The basic assumption of the superiority of solo authorship in social work needs to be rigorously questioned through strong advocacy of the multiple benefits of fair and ethical collaboration.

\section{References}

American Psychological Association. (1992). Ethical principles of psychologists and code of conduct. American Psychologist, 47, 1597-1611.

American Psychological Association. (1994). Publication Manual (4th ed.). Washington, DC: Author.

Aram, J. D., Morgan, C. D., \& Esbeck, E. S. (1971). Relation of collaborative interpersonal relationships to individual satisfaction and organizational performance. Administrative Science Quarterly, 16, 289-296. 
Austin, A., \& Baldwin, R. (1991). Faculty Collaboration: Enhancing the Quality of Scholarship and Teaching. (ASHE-ERIC Higher Education Report No. 7). Washington, DC: The George Washington University, School of Education and Human Development.

Bayer, A., \& Smart, J. (1991). Career publication patterns and collaborative "styles" in American Academic Science. Journal of Higher Education, 62(6), 613-636.

Bonosky, N. (1995). Boundary violations in social work supervision: Clinical, education, and legal implications. The Clinical Supervisor, 13(2), 79-95.

Congress, E. (1996). Dual relationships in academia: Dilemmas for social work educators. Journal of Social Work Education, 32(3), 329-338.

Endersby, J.W. (1996). Collaborative research in the social sciences: Multiple authorship and publication credit. Social Science Quarterly, 77(2), 375-392.

Fine, M., \& Kurdek, L. (1993). Reflections on determining authorship credit and authorship order on facultystudent collaborations. American Psychologist, 48(11), 1141-1147.

Floyd, S., Schroeder, D., \& Finn, D. (1994). Only if I'm first author: Conflict over credit in management scholarship. Academy of Management Journal, 37, 734-747.

Fox, M. F., \& Favor, C. A. (1984). Independence and cooperation in research: The motivations and costs of collaboration. Journal of Higher Education, 55(3), 347-359.

Gelman, S. R., \& Gibelman, M. (1999). A quest for citations? An analysis of and commentary on the trend toward multiple authorship. Journal of Social Work Education, 35(2), 203-213.

Gibelman, M., \& Gelman, S. R. (1999). Who's the author: Ethical issues in publishing. Arete, 23(1), 77-88.

Gordon, M. D. (1980). A critical assessment of inferred relations between multiple authorship, scientific collaboration, the production of papers, and their acceptance for publication. Scientometrics, 2, 193-201.

Kagel, J., \& Giebelhausen, P. (1994). Dual relationships and professional boundaries. Social Work, 39(2), 213-220.

Krysik, J., \& Nichols-Casebolt, A. (1994). Women authors in social work journals: A research note. Social Work Research, 18(3), 186-193.

Leatherman, C. (1997, July 18). Should dog walking and house sitting be required for a Ph.D.? The Chronicle of Higher Education, A10-A11.

McCullagh, J.G. (1988). Trends in publication: A study of contributors to the School Social Work Journal. School Social Work Journal, 13(1), 4-12.

National Association of Social Workers. (1996). NASW Code of Ethics. Washington DC: author.

Netting, F., \& Nichols-Casebolt, A. (1997). Authorship and collaborations: Preparing the next generation of social work scholars. Journal of Social Work Education, 33(3), 555-564.

Over, R. (1982). Collaborative research and publication in psychology. American Psychologist, 37(9), 9961001.

Presser, S. (1980). Collaboration and the quality of research. Social Studies of Science, 10, 95-101.

Task Force on Social Work Research. (1991). Building social work knowledge for effective services and policies: A plan for research development. Washington, D.C.: National Institute of Mental Health.

Wright, C., \& Wright, S. (1987). The role of mentors in the career development of young professionals. Family Relations, 36, 204-208.

Zook, A. (1987). Trend toward multiple authorship: Update and extension. Journal of Counseling Psychology, 34, 77-79.

\section{Author's Note:}

Address correspondence to: Angeline Barretta-Herman, Ph.D., School of Social Work, College of St. Catherine/University of St. Thomas, 2115 Summit Avenue, St. Paul, Minnesota 55105 USA. 